\title{
Design of Model of VANET-based Parking Space Information Distribution
}

\author{
Gang Cheng \\ Communication \& Network Center, Lanzhou University, Lanzhou, 730000, China \\ Email: cg@Izu.edu.cn
}

Keywords: Vehicle Ad Hoc Network; Parking Space Information; Credit Incentive Mechanism

\begin{abstract}
As a variety of smart mobile terminals are in high-speed development, Vehicle Ad Hoc Network (VANET) reveals its huge potential of application in road traffic control and parking space information distribution and acquisition by VANET is one of its important applications. In this paper, a model of distributing parking space information by Ad Hoc Network in cities is presented: it distributes parking space information by combining V2V (vehicle to vehicle) and V2I (vehicle to infrastructure) communications and introduces credit incentive mechanism to defend against effects of greedy nodes on network security and stability. V2V communication and V2I communication complement and cooperate with each other to cover majority of parking space resources in cities and credit incentive mechanism solves inevitable problems of spoofing and manipulation in routing protocol.
\end{abstract}

\section{Introduction}

In modern society, network technology and urban road traffic are neck and neck at a high speed in development. As a result, communication of traffic information by vehicle network becomes a hot issue in network technology application research. Vehicle Ad Hoc Network is applied to understand road condition, adjust driving route, receive warning information of other nodes in the network and coordinate in vehicle speed so as to prevent accident of rear-end collision, etc. in an accident [1].

Parking space navigation is an important application in Vehicle Ad Hoc Network. Statistics in the literature [2] show that Schwabing, a German city, annually spends EUR 20 million in finding parking spaces and 150,000 hours in waiting in total. Therefore, in urban traffic environment, it saves plenty of time and energy consumption by applying information in the Vehicle Ad Hoc Network to quickly location unoccupied parking spaces.

According to the basic idea in Vehicle Ad Hoc Network, vehicles in some range of communication exchange their vehicle speed, location and other information and data sensed by vehicle sensor and automatically connect and establish a mobile network. The whole Vehicle Ad Hoc Network is divided to two parts: vehicle to vehicle (V2V) and vehicle to infrastructure (V2I). In this paper, concerning characteristics and technical difficulties in the Vehicle Ad Hoc Network, the mode of Ad Hoc network in V2V communications is primarily discussed.

\section{Model of Parking Space Information Distribution}

\section{Overall design.}

The model of parking space information distribution designed in this paper is briefly generalized as shown in Figure 1 in which all components in the system have the following functions: parking space charge terminal: each parking space is equipped with parking space charge terminal and information of whether the parking space is unoccupied is supplied to infrastructure.

Infrastructure: it is assumed in this paper that a small number of fixed infrastructures for collecting and distributing parking space information exist in cities. Each infrastructure collects information of parking space occupation (and charge terminal communication) in its range of jurisdiction, systemizes and summarizes to respond to vehicle request and supplies situation of parking space occupation to vehicles. 


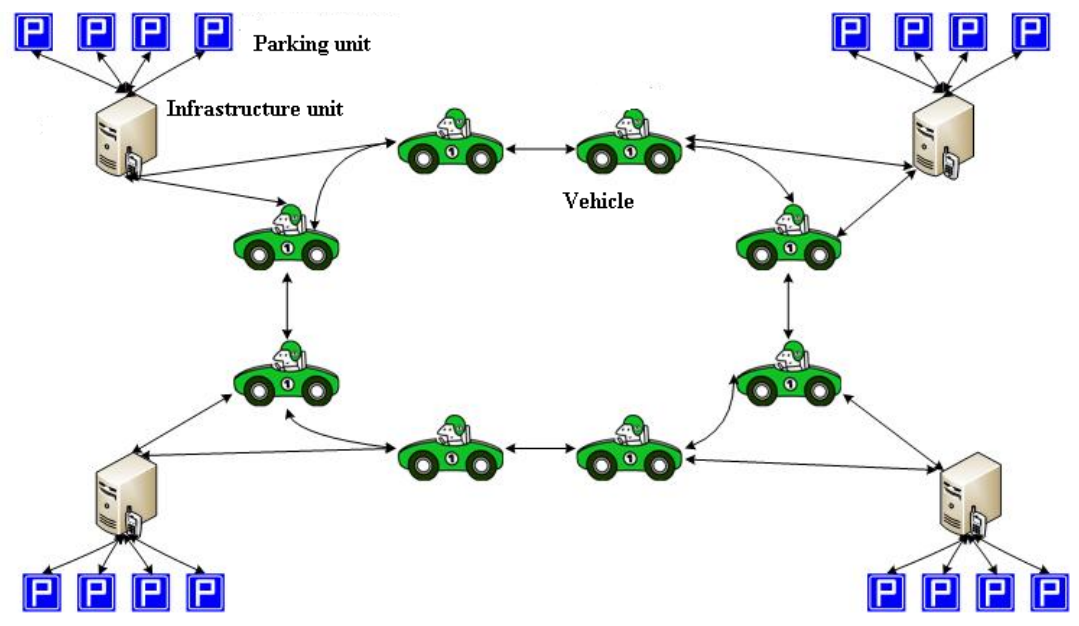

Fig1 Distribution Model of Parking Places Information

Motor vehicle: in order to reduce system sophistication, it is assumed in this paper that each motor vehicle is equipped with sensor and GPS. A motor vehicle node in the system acquires surrounding parking space information in two ways: firstly, it spontaneously sends request to neighboring infrastructures and acquires unoccupied parking space information near the destination; secondly, it reactively receives and forwards broadcast information periodically sent by other vehicles in the network. Meanwhile, motor vehicle node posts parking space information in two ways: firstly, it sends information to infrastructure and updates state of parking space as soon as it leaves some parking space; secondly, it sends broadcast information to nearby motor vehicle nodes.

\section{Parking space information.}

The information sent by each parking space charge terminal, as a parking space information unit, is defined to be $\mathrm{p}=\{\mathrm{id}$,flag $\}$.

id: each parking space is assigned with one identification number that is applied to correspond to specific location of the parking space in the infrastructure sheet;

flag: service flag bit, classified to two states: unoccupied and occupied.

Infrastructure summarizes all parking space information units in its range of jurisdiction, finds specific locations of corresponding unoccupied parking spaces in its own data sheet based on id in the parking space information unit, calculates and summarizes the probability of non-occupation of parking spaces upon arrival of the vehicle at its destination according to current location, vehicle speed and destination sent by the vehicle to parking space information block and recommends the top three parking spaces to motor vehicle node based on probability decrease. The parking space information block is defined as:

$\mathrm{pg}=\{\{$ id1,loc1, $\quad$ probability1 $\},\{$ id2,loc2, probability2 $\},\{\mathrm{id} 3, \operatorname{loc} 3, \quad$ probability3 $\}\}$ whose parameters are as follows:

id: identification number of parking space recommended by infrastructure;

loc: specific geographical location of unoccupied parking space;

probability: probability of non-occupation of parking space upon arrival of vehicle calculated by infrastructure.

\section{Parking space information stream.}

Since the mode of collaboration of V2V and V2I communications is applied in this paper, motor vehicle node may not only spontaneously request infrastructure to recommend available parking spaces (parking space information block) but also acquire unoccupied parking space information (parking space information unit) forwarded by vehicles or periodically broadcast by charge terminal. Two information streams in the system co-exist and complement each other.

1) V2I (vehicle-to-infrastructure) communication

It is assumed in this paper that there are a small number of fixed infrastructures for collecting and distributing parking space information in cities. Each infrastructure has some coverage due to information processing capacity in strength difference and restriction in some physical properties, 
defined to be a circle centered by infrastructure in specific radius. Each infrastructure is in charge of collecting and distributing parking space information in its own coverage (by charge terminal). The physical topology of V2I communication is shown in Figure 2:

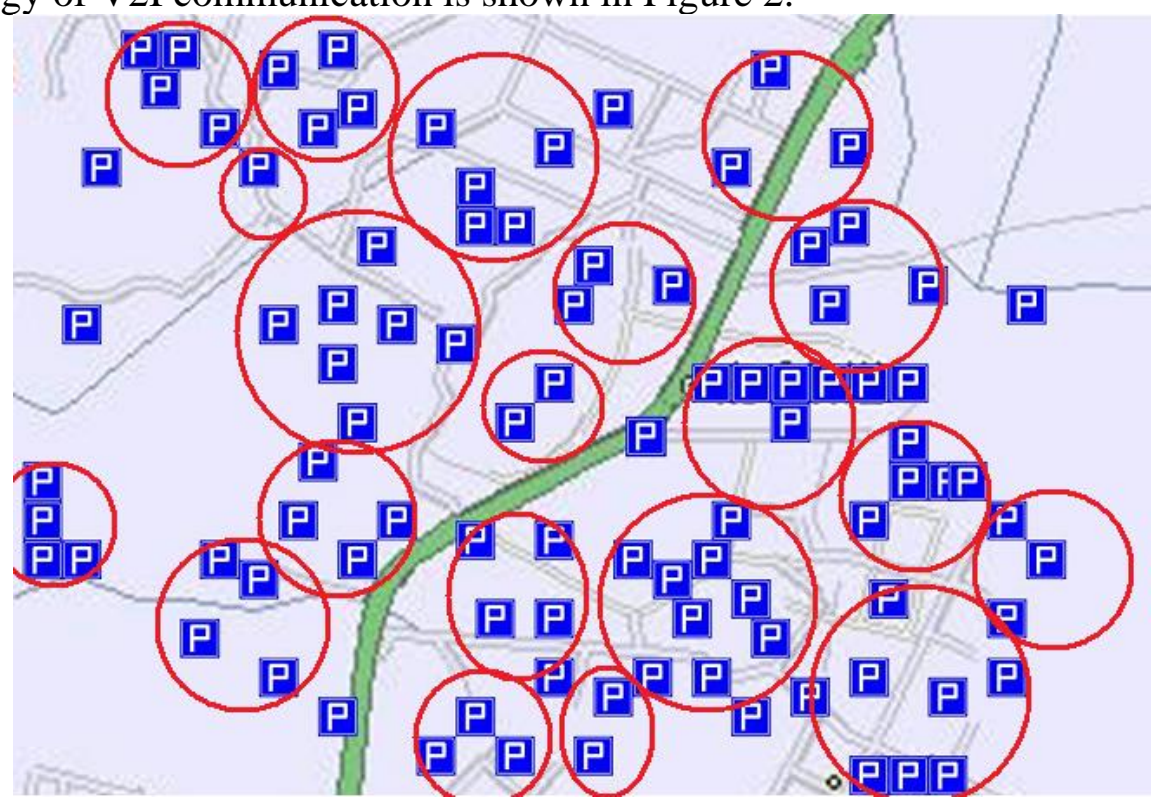

Fig2 The Topology of V2I Communication

Based on the above topology, each infrastructure masters occupation of parking spaces in its range of jurisdiction. A vehicle supplies its current driving location and vehicle speed (supplied by GPS) and destination (supplied by driver) to an infrastructure as soon as it enters range of control of the infrastructure. The infrastructure posts unoccupied parking space information near the vehicle's destination to the driver for selection. The process of V2I communication is shown in Figure 3:

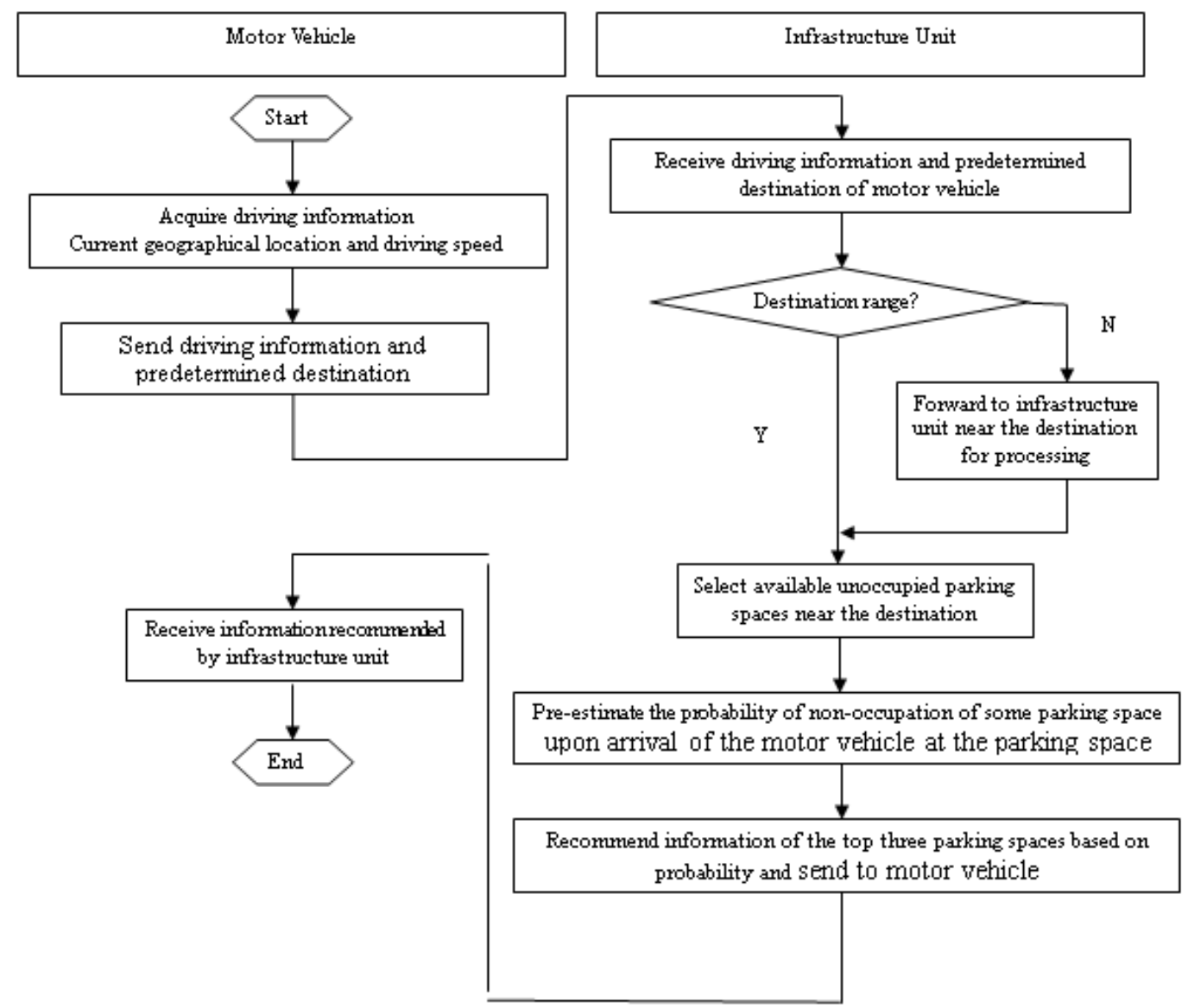

Fig3 The Process of V2I communication 
Where a motor vehicle is required to spontaneously request infrastructure to send parking space information, it acquires its own driving-related information first of all: GPS supplies current specific geographical location, vehicle module supplies current driving speed and driver supplies specific location of predetermined destination. And the information is packaged and sent to infrastructure. The infrastructure finds available unoccupied parking spaces nearby based on the destination's information, calculates the probability of non-occupation of some parking space upon arrival of the vehicle at the parking space based on vehicle's driving state and other requests, and recommends the top three unoccupied parking spaces based on the probability to driver for selection.

Where destination supplied by a motor vehicle is not covered by current infrastructure, the infrastructure forwards request information to infrastructure covering the destination by nearby infrastructure. It is bound to find destination's infrastructure in the network by finite step forwarding. Moreover, normally, as a motor vehicle is approaching its destination, it is bound to find an infrastructure for immediately acquiring destination's parking space information without forwarding in an area closer to the destination by periodically sending request information to infrastructures on its way.

2) V2V (vehicle-to- vehicle) communication

It is known from the above topology that infrastructure fails to cover all parking space information and that some parking spaces are in sparser areas where it is not economic to establish an infrastructure. Therefore, vehicle infrastructure is in charge of detecting and updating state of parking spaces in the areas and broadcasting to nearby nodes.

In initial state, memory in a vehicle infrastructure does not contain any parking space information. As a vehicle is driven, its vehicle infrastructure receives parking space information from infrastructure, charge terminal or other vehicle infrastructures and broadcasts to nearby nodes in some period.

\section{Security}

In a mobile Ad Hoc network, whether network is in normal operation greatly depends upon cooperation of nodes. If there is any uncooperative node or any node that enjoys services supplied by network without supplying services, it will gravely influence normal operation of network. The strategy of coping with uncooperative nodes is similar to that against greedy nodes-introduction of incentive and credit mechanism.

The message information distributed by vehicles in the system refers to parking space information, which is important information closely related to interests of each node (whether to find available parking spaces as soon as possible). Therefore, the following two issues of specificity which may threaten system security should be specially focused in operation: spoofing of non-motor vehicle node and message manipulation of greedy motor vehicle.

In order to seek commercial interest, a parking lot may disguise itself as a motor vehicle node to send spoofing information that some vehicle has departed from some parking space so as to enhance idleness of parking space in the parking lot to attract vehicle owners. In order to avoid the problem of security resulting from spoofing, message identification mechanism is introduced [2,3]. If a shared secret key $\mathrm{K}$ exists between two nodes, both nodes will generate and test message identification code by calculating message hash value hK (Message) with secret key K in which the $\mathrm{h}$ will be a one-way hash function. Such a message identification mechanism is good for small calculated amount and simple process of generation and verification.

Vehicle infrastructure manufacturer equips each vehicle infrastructure with digital certificate to certify legitimate identity of the vehicle infrastructure in the system. Administration of traffic control equips each infrastructure with digital certificate. In the process of parking space information message transmission, each node (including motor vehicles and infrastructures) undergoes secret key verification, first of all, and information sent by node verified by secret key is accepted. Otherwise, it will be discarded.

Such a motor vehicle node may exist in the system: in order to enhance its probability of 
acquiring unoccupied parking space information as soon as possible, it makes malicious manipulation while forwarding message information to revise state of originally unoccupied parking space to be occupied or revise the probability value in the parking space information block to be a very low value so as to deceive nodes nearby its destination to select other unoccupied parking spaces.

Since such a manipulation is not concerning routing message transmitted in the network, it is advised to introduce credit incentive mechanism to avoid such a problem instead of designing or selecting algorithm based on specificity of routing protocol. Specifically, it is described as follows:

Form of credit: each vehicle module has its own initial credit point.

Form of surveillance: exceptions of neighboring nodes are monitored by motor vehicle node, for instance, too low probability value in the parking space information block forwarded by some node, and corresponding credits are updated based on the exceptions. Where a node has evidence to believe that some monitored object is exceptional, it sends a piece of ALARM message to notify other nodes whose monitoring modules hand it over to trust management module for processing as soon as they directly or indirectly receive the ALARM message. The trust management module, first of all, evaluates the credibility of ALARM. If confirming that it is highly credible, it will notify credit system of updating credit value of the reported object. If the credit value is out of security threshold, such a situation will be responded to route management module.

Form of reward and punishment: nodes in high credit may be processed in some priority in the request queue of infrastructures to enable the cooperative nodes to more quickly acquire information; once credit of some vehicle infrastructure declines to some unacceptable bottom line, the node will be isolated and required of undergoing some punishment by means of administrative management to rejoin the system.

\section{Conclusions}

In this paper, a model of distributing parking space information by Ad Hoc network in cities is presented: it distributes parking space information by combining V2V and V2I communications and introduces credit incentive mechanism to defend against effects of greedy nodes on network security and stability. If system in this paper is applied to a specific traffic environment, solving an optimal time interval will be an issue worth discussing. In view of rapid mobility of nodes in VANET, broadcast time interval will have distinct effects on system performance [4]. The dispatch strategy of infrastructure responding to vehicle request: since multiple vehicles simultaneously send requests to infrastructure, it is not feasible to purely apply traditional first-come-first-service strategy. In this paper, credit incentive mechanism is introduced to each vehicle module. Therefore, node priority should be taken into account in infrastructure’s request response dispatch [5].

\section{References}

[1] Ziyuan Wang,Lars Kulik,Kotagiri Ramamohanarao.Proactive Traffic Merging Strategies for Sensor-Enabled Cars.VANET’07, September 10, 2007, Montréal, Québec, Canada.

[2] LIU Pei-chao, YANG Hao, ZHOU Xi. Research of Wireless Ad Hoc Network Security Authentication,

Computer Knowledge and Technology, 2009.

[3] Liang HONG. Research on Secure MANET Routing Protocols: dissertation, Huazhong University of Science and Technology.2006

[4] Stephan Eichler, Christoph Schroth.Car-to-Car Communication. Institute of Communication Networks, Technische University, Germany.

[5] Yang Zhang, Jing Zhao, Guohong Cao. On scheduling vehicle-roadside data access.VANET’07, September 10, 2007, Montréal, Québec, Canada. 